\title{
Light Path Model of Fiber Optic Liquid Level Sensor Considering Residual Liquid Film on the Wall
}

\author{
Zhijun Zhang and Shiwei Zhang \\ School of Mechanical Engineering and Automation, Northeastern University, Shenyang 110004, China \\ Correspondence should be addressed to Zhijun Zhang; zhjzhang@mail.neu.edu.cn
}

Received 11 December 2014; Revised 25 February 2015; Accepted 27 February 2015

Academic Editor: Dong-ning Wang

Copyright (C) 2015 Z. Zhang and S. Zhang. This is an open access article distributed under the Creative Commons Attribution License, which permits unrestricted use, distribution, and reproduction in any medium, provided the original work is properly cited.

The working principle of the refractive-type fiber optic liquid level sensor is analyzed in detail based on the light refraction principle. The optic path models are developed in consideration of common simplification and the residual liquid film on the glass tube wall. The calculating formulae for the model are derived, constraint conditions are obtained, influencing factors are discussed, and the scopes and skills of application are analyzed through instance simulations. The research results are useful in directing the correct usage of the fiber optic liquid level sensor, especially in special cases, such as those involving viscous liquid in the glass tube monitoring.

\section{Introduction}

Liquid level monitoring is necessary in the industry and in the scientific research field [1]. The designs of liquid level sensors indicate the preset height of liquid levels, including magnetic [2], vibrant, capacitive [3], microwave [4], ultrasonic [5], and photoelectric liquid level sensor $[6,7]$. The traditional glass tube liquid level gauge is widely utilized in simple structures for clear indication and visual observation $[8,9]$. When a glass tube liquid level gauge is combined with a fiber optic sensor, a type of fiber optic liquid level sensor is formed. It includes the optical emission fiber, optical acceptance fiber, light source, optical modulator, amplifier, and glass tube liquid level gauge $[10,11]$. The advantage of this sensor is that it can achieve genuine noncontact liquid level monitoring. The additional sensor elements, such as float or fiber, are not required to to be set in the liquid [12-16]. The micro size of the optic fiber enhances the accuracy of liquid level monitoring. The transformation of old equipment, which consists only of the glass tube liquid level gauge and which works by relying on the observations of the naked eye, into automatic liquid level monitoring by adding fiber optic sensors to the outside of the glass tube is convenient. The fiber optic liquid level sensor has the characteristics of fast response, corrosion resistance, electrical insulation, and small volume as well as the ability to easily compose the remote monitoring network with the fiber optic transmission system. Coupled with the high sensitivity of the electronic amplifier, this sensor possesses outstanding advantages in terms of antielectromagnetic interference capability and high sensitivity $[17,18]$.

As the advantage of fiber optic liquid level sensor, the study, and application are wide, a noncontact fiber optic liquid level sensor based on the phenomenon of reflective concept is presented [19]. The device consists of fiber optic transmitter, fiber optic probe, floating reflector, photodiode detector, and digital multimeter. A simple intensity-modulated fiber optic sensor is developed based on frustrated total internal reflection effect caused by refractive index change of a medium surrounding an optical fiber [20]. An optical fiber sensor liquid level based on light intensity modulation and such modulation results from alteration of total internal reflection into partial reflection at the interface are reported [21]. A liquid level sensor based on the refractive-index sensitivity of long-period fiber optic gratings is proposed and demonstrated. The form of the transmission spectrum of the long-period grating is dependent on the fraction of the length of the long-period grating that is surrounded by the liquid [7]. A fiber optic level sensor with special microoptical refractometric transducer is designed to measure the level 
of liquid propane-butane in a relatively short range $(60 \mathrm{~cm})$ in the top part of storage tanks at oil refineries [22]. A micro change in level using plastic optical fiber sensor is demonstrated [23]. This sensor operates on light intensity modulation and such modulation results due to displacement of the reflector floating on the liquid. An experimental realization of a simple all-fiber optic sensor for liquid level measurement is demonstrated. It is an intensity-modulated on-off switching sensor whose operating principle is based on the frustrated total internal reflection effect caused by the refractive-index change of the surrounding medium [24]. An intensity-based fiber optic liquid level sensor for point measurement is described. The sensing principle is based on the total internal reflection of light, which is disturbed by contact with a liquid [25]. A simple and compact reflective liquid level sensor based on modes conversion in the thincore fiber incorporating one tilted fiber Bragg grating (TFBG) is proposed and experimentally demonstrated [26]. A new and simple fiber optic liquid level sensor based on light intensity modulation was presented when rising and falling mode of liquid level causes coupling optical path distance variation between two optical fibers [27].

A fiber optic liquid level sensor that is conducted by a combination of optical interferometer and lever principle is presented [12]. Its sensing unit is a Mach-Zehnder interferometer (MZI). The measuring equipment is composed of a rotatable lever and a fixed link. A fiber optic liquid level sensor based on multimode interference (MMI) effects is proposed and demonstrated [13, 17]. The MMI and selfimage effects can be effectively applied for multiplexed liquid level sensing, because the natural response as a band-pass filter for each sensor is clearly distinct from each other, in the case for several sensors working at the same time. A simple and low-cost discrete liquid level measurement system which consists of a group of plastic optical fiber segments, which are aligned coaxially and spaced equally, is present [28]. A novel optical liquid level sensor based on polarizationmaintaining fiber (PMF) modal interferometer with waist enlarge splicing is proposed [29]. The design and packaging of simple, small, and low-cost sensor heads, used for continuous liquid level measurement using uniformly thinned (etched) optical fiber Bragg grating (FBG) are proposed [30]. An asymmetric Fabry-Perot fiber optic pressure sensor is capable of precisely measuring liquid levels [31]. They are directly proportional to pressures that can modulate cavity length of Fabry-Perot cavity. An efficient approach to develop largerange liquid level sensors based on an extrinsic Fabry-Perot optical fiber interferometer with an all-fused-silica structure and $\mathrm{CO}_{2}$ laser heating fusion bonding technology is also presented [32].

Although the principle of the refracting-type fiber optic liquid level sensor is simple and clear, the method and rules for its application, such as the arrangement of both emission and acceptance optical fibers, are quite complex. In practice, the position and angle of the optical fibers are determined by driftless tests in most cases because of the lack of quantitative theoretical basis. However, blind search does not always work, and theoretical analysis is necessary in special cases of applications.

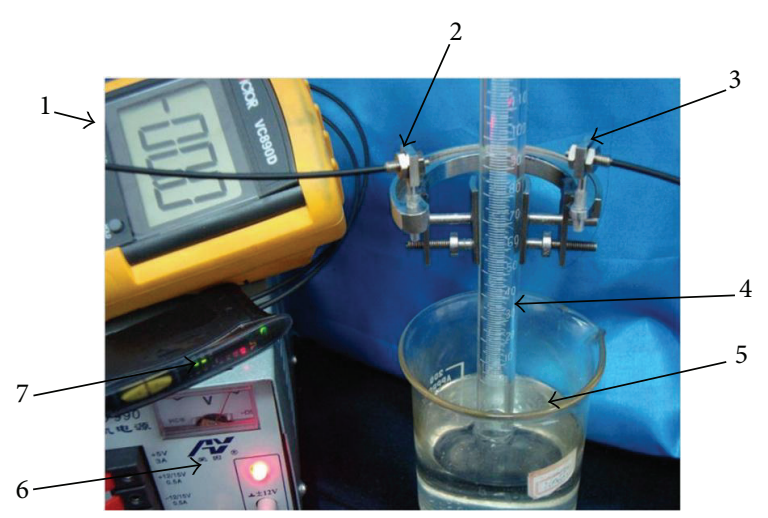

FIGURE 1: The photograph of fiber optic liquid level sensor in present study.

\section{Problem Description}

The study in present paper is from the practical problem encountered when they developed the "automatic vapor vacuum pump performance measurement system" patented in China (Chinese Patent: ZL200910012545.3) [33]. According to the performance measuring method of vapor vacuum pump which is recommended by both the international standard ISO 1607-1 (1993) and the Chinese standard GB/T 19955.1 (2005), the measurement of volume rate of flow (pumping speed) needs to monitor the liquid level in a series of glass tubes with given diameter. In the experiment, the liquid level is forced to rise and drop several times between the two preset heights which is $20 \mathrm{~cm}$. The change of liquid level is because of the change of pressure on the top of liquid. The inner diameters of the glass tubes in present study are $1.5 \mathrm{~mm}, 3 \mathrm{~mm}$, and $5 \mathrm{~mm}$ according to above standard. The liquid medium in the tube is a type of industry transformer oil with lower saturation vapor pressure limiting oil pollution for vapor vacuum pump. The oil is transparent and has higher viscosity. In order to realize the automatic test, the fiber liquid level sensors are the best method as above mentioned. The two fiber optic liquid level sensors are set and provide the responsive signals. The device photograph of fiber optic liquid level device is show in Figure 1.

The refracting-type fiber optic liquid level sensor was developed based on the principle of light refraction. The incidence detecting light beam ( $A B$ in Figure 2) from the optical emission fiber arrives at the outer surface of the glass tube obliquely and passes through the glass tube sinuously. The light beam $\left(G_{T} H_{T}\right.$ or $G_{D} H_{D}$ in Figure 2$)$ is then received by the optical acceptance fiber, which is located on the other side of the glass tube at a slanted angle. The optical path is a zigzag line across the glass tube because the light beam is refracted at each interface between two transparent media with different refractive indices. The light path through the liquid $\left(A B C F_{D} G_{D} H_{D}\right.$ in Figure 2$)$ is different from the path through the gas $\left(A B C F_{T} G_{T} H_{T}\right.$ in Figure 2) because of the difference in the refractive indices of the liquid and gas inside the glass tube. Therefore, the position and angle of the emergent detecting light beam passing through the liquid are 


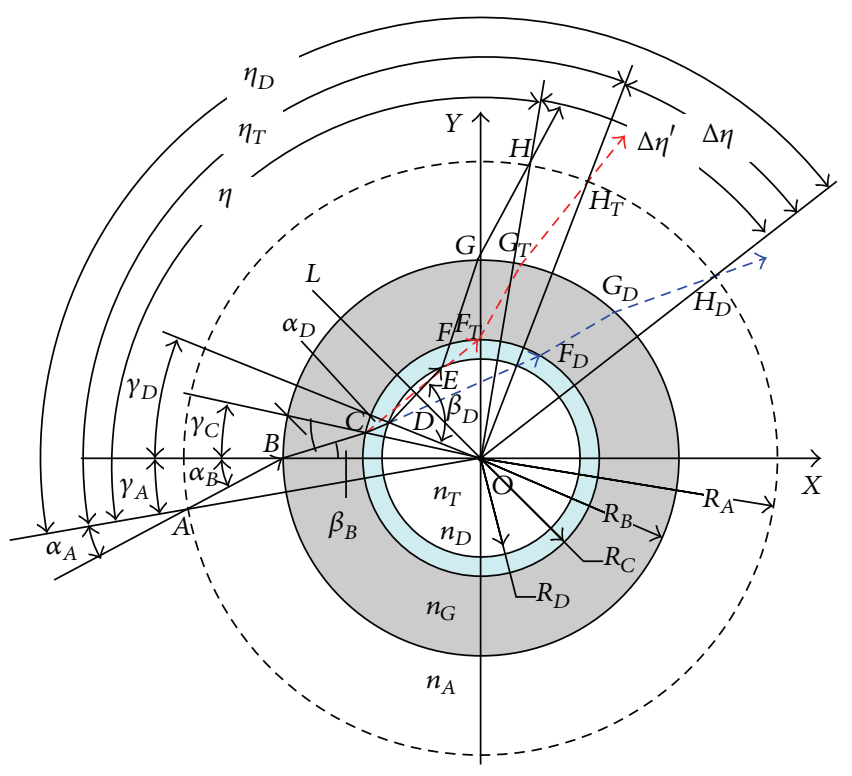

FIGURE 2: Light path models of three conditions: gas in glass tube $\left(A B C F_{T} G_{T} H_{T}\right)$, liquid medium in glass tube $\left(A B C F_{D} G_{D} H_{D}\right)$, and residual liquid film on the glass wall ( $A B C D E F G H)$.

different from those of the beam passing through the gas. If the optical acceptance fiber is arranged at a position and angle corresponding to the emergent detecting light beam passing through the liquid with the possibility of receiving the light signal, the emergent detecting light beam passing through the gas will not be received and vice versa. Thus, the refractingtype fiber optic liquid level sensor works well when the liquid is good and transparent.

However, the optical acceptance fiber cannot frequently receive the emergent light in time at expected emergent point $\left(G_{T} H_{T}\right.$ in Figure 2) because the light path is changed to $G H$ as in Figure 2. The change is the residual oil film on the tube wall. It is the medium changing the light path. The thickness of oil is changed with the time due to which $G H$ is changed. The oil film effect is not known well until now. The theory study would clarify the detail information and maybe would guide the application of sensors.

In this paper, the light path model considering the residual liquid film is established. The derivation and analysis are performed to meet the requirements for theoretical research provided above. The working mechanism of the refractingtype fiber optic liquid level sensor with glass liquid level is discovered.

\section{Review of Simplified Model of Optical Path}

The simplified model of optical path calculation [33] is shown with dotted line in Figure 1. It is the cross-section of the glass tube. Glass tube center $O$ is considered to be the coordinate origin; a rectangular coordinate system $X O Y$ is established. The darker part is the cylindrical wall of the glass tube, the outer radius of which is $R_{B}$ and the inner radius of which is $R_{C}$. At the circle with radius $R_{A}$, point $A$ is the incident point of the light and point $H$ is the emergent point of the light. Lastly, the original positioning parameter of the light path is set. The coordinate of incident point $B$ is selected as the positioning point of the light path. In the rectangular coordinate system $X O Y$, the coordinate of point $B$ is $\left(x_{B}=\right.$ $-R_{B}, y_{B}=0$ ).

The parameters and symbols of the various angles in Figure 1 are defined as follows.

(1) Azimuth: the angle between the negative $X$ axis of the coordinate and the normal line at each turning point $(A B C F G H)$ in the light path, denoted by $\gamma$ with a subscript, such as $\gamma_{A}, \gamma_{B}$, and $\gamma_{C}$.

(2) Incidence angle: the angle between the incident light and the normal line at each turning point $(A B C F G H)$ in the light path, denoted by $\alpha$ with a subscript, such as $\alpha_{A}, \alpha_{B}$, and $\alpha_{C}$.

(3) Refraction angle: the angle between the refraction light and the normal line at each turning point $(A B C F G H)$ in the light path, denoted by $\beta$ with a subscript, such as $\beta_{B}$; the refraction angle at point $C$ is especially distinguished when the refractive indices of the media in the tube are different, such as with $\beta_{C T}$ and $\beta_{C D}$.

(4) The total deflection angle: also called total acceptance angle, this angle is the azimuth difference between light incident point $A$ and emergent point $H_{D}$ or $H_{T}$; when the optically denser medium $n_{D}$ is in the tube, $\eta_{D}$ is noted as the total deflection angle; when the optically thinner medium $n_{T}$ is in the tube, $\eta_{T}$ is noted as the total deflection angle; the difference is expressed as the deflection angle difference denoted by $\Delta \eta$.

Four kinds of optical media divided by the optical refractive index are involved in the glass tube liquid level gauge. Their refractive indices are denoted by $n_{A}, n_{G}, n_{D}$, and $n_{T}$ in outward to inward turns. The medium outside the tube that $n_{A}$ stands for is generally air; therefore, $n_{A} \approx 1$. However, this relationship is not directly used to simplify the formulae in the following derivation and calculation. $n_{G}$ stands for the refraction index of the glass tube. Two kinds of media are present in the tube, and their refraction indices must be different, which is the physical basis of the method introduced in this paper. The medium with the larger refractive index is called the optically denser medium denoted by $n_{D}$; the medium with the less refractive index is called the optically thinner medium denoted by $n_{T}$. Both $n_{D}$ and $n_{T}$ may be less than $n_{G}$. The optically denser medium is typically a liquid, whereas the optically thinner medium is typically a gas.

The detecting light emitted from the emission fiber goes through this plane. Incident light $A B$ enters the tube at angle $\alpha_{B}$. The outer surface is normal at point $B$. When gas is in the tube, the light refracts at points $B, C, F_{T}$, and $G_{T}$ on the inside and outside surfaces of the tube wall when light passes through the tube. The light is finally received by the optical acceptance fiber at point $H_{T}$. By contrast, the refraction angle of the light changes at point $C$ on the inside of the tube wall when the tested liquid in the tube reaches the height of the light and when the refractive index changes, resulting in the change in the path followed by the light. The entire light path changes to $A B C F_{D} G_{D} H_{D}$. As point $H_{D}$ deflects from the original accepting position of point $H_{T}$, the optical 
acceptance fiber at point $H_{T}$ can no longer receive the light signal. Thus, a great contrast with regard to the existence of light at the points $H_{D}$ and $H_{T}$ is present before and after the increase in the liquid level, thereby improving the responses sensitivity especially in transparent media.

\section{Light Path Model Considering Residual Liquid Film on the Wall}

The liquid in the tube is usually characterized by high viscosity and large surface tension. The signal response when the liquid surface rises and passes the detecting light position is unaffected. However, a residual layer of liquid film appears on the glass wall when the liquid drops and merely passes the detecting light position. Thus, the incident light must first pass the liquid film to arrive at the gas area in the tube. The emergent light must also pass through the liquid film to pass the glass wall; therefore, the detecting light must pass through the liquid film twice and refract on the interface. In this period of time, the emergent light angle deviates from $\eta_{T}$ when only gas is present in the tube as predicted in the simplified model. Over time, the liquid on the wall falls automatically because of gravity, thereby making the liquid film thinner and the refraction of the detecting light smaller until the tube is full of gas and the angle position of the emergent light returns to $\eta_{T}$. The problem of residual liquid film on the wall during the falling process directly causes errors in liquid level monitoring. Therefore, special research on this phenomenon is necessary.

The light path calculation model considering the residual liquid film on the wall adopts the basic assumption and parameter definition of the previous simplified model [33]. The graph of the light path model shown with solid line in Figure 1 was established based on [33]. The only change is the addition of a layer of liquid film in the glass tube, which is a circle concentric with the inner radius of $R_{D}$. This addition causes the detecting light path in the glass tube to change from line $C F_{T}$ into three line segments $C D, D E$, and $E F$ in present study in Figure 2.

4.1. Hypothesis. (1) The outer and inner surfaces of the glass tube are standard coaxial cylindrical surfaces. The shape error is ignored.

(2) All materials involved in the light path are isotropic homogeneous transparent optical media. The detecting light in various media is linear, and the light path obeys the refraction law at the interfaces of the two kinds of media.

(3) The size of the light can be ignored; that is, it can be regarded as a geometric straight line because it is very fine relative to the tube size.

4.2. Coordinate, Azimuth, and Incident Angle of Point A. Point $A$ is the intersecting point of the incident light $y=$ $k_{B}\left(x+R_{B}\right)$ with the circle $x^{2}+y^{2}=R_{A}^{2}$ where the optical emission fiber is located. The slope of the incident light is $k_{B}=\tan \alpha_{B}$, and the coordinate of point $A$ is

$$
\begin{gathered}
x_{A}=-R_{B} \cdot\left\{\cos \alpha_{B} \cdot\left[\left(\frac{R_{A}}{R_{B}}\right)^{2}-\sin ^{2} \alpha_{B}\right]^{1 / 2}+\sin ^{2} \alpha_{B}\right\}, \\
y_{A}=R_{B} \cdot \sin \alpha_{B} \cdot\left\{\cos \alpha_{B}-\left[\left(\frac{R_{A}}{R_{B}}\right)^{2}-\sin ^{2} \alpha_{B}\right]^{1 / 2}\right\} .
\end{gathered}
$$

The azimuth of point $A$ can be expressed by the slope of line $y=f_{A} \cdot x$ as

$$
\begin{aligned}
\tan \gamma_{A} & =f_{A} \\
& =\frac{y_{A}}{x_{A}} \\
& =\frac{\sin \alpha_{B} \cdot\left\{\left[\left(R_{A} / R_{B}\right)^{2}-\sin ^{2} \alpha_{B}\right]^{1 / 2}-\cos \alpha_{B}\right\}}{\cos \alpha_{B} \cdot\left[\left(R_{A} / R_{B}\right)^{2}-\sin ^{2} \alpha_{B}\right]^{1 / 2}+\sin ^{2} \alpha_{B}} .
\end{aligned}
$$

At point $A$, the incidence angle $\alpha_{A}$ is

$$
\begin{aligned}
\tan \alpha_{A} & =\frac{k_{B}-f_{A}}{1+k_{B} f_{A}} \\
& =\frac{\sin \alpha_{B}}{\left[\left(R_{A} / R_{B}\right)^{2}-\sin ^{2} \alpha_{B}\right]^{1 / 2}} .
\end{aligned}
$$

4.3. Refraction Angle of Point $B$. At the interface of point $B$, $n_{A} \sin \alpha_{B}=n_{G} \sin \beta_{B}$ according to the refraction law. The refraction angle of point $B$ can thus be obtained as follows:

$$
\sin \beta_{B}=\frac{n_{A}}{n_{G}} \sin \alpha_{B}
$$

or

$$
\tan \beta_{B}=\frac{\left(n_{A} / n_{G}\right) \cdot \sin \alpha_{B}}{\left[1-\left(n_{A} / n_{G}\right)^{2} \cdot \sin ^{2} \alpha_{B}\right]^{1 / 2}} .
$$

4.4. Coordinate, Azimuth, and Incident Angle of Point C. Point $C$ is the intersecting point of the refracted light from point $B y=k_{C}\left(x+R_{B}\right)$ with the circle $x^{2}+y^{2}=R_{C}^{2}$ on the inner wall of the glass tube. The slope of the refracted light is $k_{C}=\tan \beta_{B}$, and the coordinate of point $C$ is

$$
\begin{gathered}
x_{C}=-R_{B} \cdot\left\{\cos \beta_{B} \cdot\left[\left(R_{C} / R_{B}\right)^{2}-\sin ^{2} \beta_{B}\right]^{1 / 2}+\sin ^{2} \beta_{B}\right\}, \\
y_{C}=R_{B} \cdot \sin \beta_{B} \cdot\left\{\cos \beta_{B}-\left[\left(R_{C} / R_{B}\right)^{2}-\sin ^{2} \beta_{B}\right]^{1 / 2}\right\} .
\end{gathered}
$$


The azimuth of point $C$ can be expressed by the slope of line $y=f_{C} x$ as

$$
\begin{aligned}
\tan \gamma_{C}= & f_{C} \\
= & -\frac{y_{C}}{x_{C}} \\
= & \left(\sin \alpha_{B}\right. \\
& \cdot\left\{\left[\left(\frac{n_{G}}{n_{A}}\right)^{2}-\sin ^{2} \alpha_{B}\right]^{1 / 2}\right. \\
& \left.\left.-\left[\left(\frac{R_{C} n_{G}}{R_{B} n_{A}}\right)^{2}-\sin ^{2} \alpha_{B}\right]^{1 / 2}\right\}\right) \\
& \cdot\left[\left(\frac{n_{G}}{n_{A}}\right)^{2}-\sin ^{2} \alpha_{B}\right]^{1 / 2} \\
& \left.\cdot\left[\left(\frac{R_{C} n_{G}}{R_{B} n_{A}}\right)^{2}-\sin ^{2} \alpha_{B}\right]^{1 / 2}+\sin ^{2} \alpha_{B}\right)^{-1} .
\end{aligned}
$$

At point $C$, the incidence angle $\alpha_{C}$ is

or

$$
\begin{aligned}
\tan \alpha_{C} & =\frac{k_{C}-f_{C}}{1+k_{C} f_{C}} \\
& =\frac{\sin \alpha_{B}}{\left[\left(R_{C} n_{G} / R_{B} n_{A}\right)^{2}-\sin ^{2} \alpha_{B}\right]^{1 / 2}}
\end{aligned}
$$

$$
\sin \alpha_{C}=\frac{R_{B} n_{A}}{R_{C} n_{G}} \sin \alpha_{B}
$$

4.5. Refraction Angle of Point C. The two kinds of media with different refractive indices in the tube correspond to two different refraction angles at point $C$; therefore, the light path after point $C$ also has two possible paths. When the refractive index of the medium in the tube is $n_{T}, n_{G} \sin \alpha_{C}=n_{T} \sin \beta_{C T}$ according to the refraction law and (8). The calculation formula of the refraction angle at point $C$ is

or

$$
\sin \beta_{C T}=\frac{R_{B} n_{A}}{R_{C} n_{T}} \sin \alpha_{B}
$$

$$
\tan \beta_{C T}=\frac{\sin \alpha_{B}}{\left[\left(R_{C} n_{T} / R_{B} n_{A}\right)^{2}-\sin ^{2} \alpha_{B}\right]^{1 / 2}} .
$$

When the refractive index of the medium in the tube becomes $n_{D}$, the refraction angle at point $C$ as calculated by the refraction law and (8) is

or

$$
\sin \beta_{C D}=\frac{R_{B} n_{A}}{R_{C} n_{D}} \sin \alpha_{B}
$$

$$
\tan \beta_{C D}=\frac{\sin \alpha_{B}}{\left[\left(R_{C} n_{D} / R_{B} n_{A}\right)^{2}-\sin ^{2} \alpha_{B}\right]^{1 / 2}}
$$

4.6. Coordinate, Azimuth, Incident, and Refraction Angles of Point $D$. Point $D$ is the intersecting point of refraction line $y=k_{D}\left(x-x_{C}\right)+y_{C}$ from point $C$ and inner surface circle $x^{2}+y^{2}=R_{D}^{2}$ of the liquid film. The slope of the refraction line is $k_{D}=\tan \left(\beta_{C D}-\gamma_{C}\right)$, the radius of the circle is $R_{D}=R_{C}-\delta$, and the thickness of the liquid film is $\delta$.

The coordinate of point $D$ in the XOY coordinates can be obtained by the following simplification:

$$
\begin{aligned}
x_{D}= & -\sin \left(\beta_{C D}-\gamma_{C}\right) \cdot \sin \beta_{C D} \cdot R_{C}-\cos \left(\beta_{C D}-\gamma_{C}\right) \\
& \cdot\left[R_{D}^{2}-\sin ^{2} \beta_{C D} \cdot R_{C}^{2}\right]^{1 / 2}, \\
y_{D}= & \cos \left(\beta_{C D}-\gamma_{C}\right) \cdot \sin \beta_{C D} \cdot R_{C}-\sin \left(\beta_{C D}-\gamma_{C}\right) \\
& \cdot\left[R_{D}^{2}-\sin ^{2} \beta_{C D} \cdot R_{C}^{2}\right]^{1 / 2} .
\end{aligned}
$$

The same applies to the relationship as follows:

$$
y_{D}-k_{D} x_{D}=y_{C}-k_{D} x_{C}
$$

Azimuth $\gamma_{D}$ of point $D$ is expressed by the slope of line $y=f_{D} x$ as

$$
\begin{aligned}
& \tan \gamma_{D} \\
& =-f_{D} \\
& =-\frac{y_{D}}{x_{D}} \\
& =\frac{\sin \beta_{C D}-\tan \left(\beta_{C D}-\gamma_{C}\right) \cdot\left[\left(R_{D} / R_{C}\right)^{2}-\sin ^{2} \beta_{C D}\right]^{1 / 2}}{\tan \left(\beta_{C D}-\gamma_{C}\right) \cdot \sin \beta_{C D}+\left[\left(R_{D} / R_{C}\right)^{2}-\sin ^{2} \beta_{C D}\right]^{1 / 2}} .
\end{aligned}
$$

The incidence angle $\alpha_{D}$ of point $D$ is determined by the following equation:

$$
\begin{aligned}
\tan \alpha_{D} & =\frac{k_{D}-f_{D}}{1+k_{D} f_{D}} \\
& =\frac{k_{D} x_{D}-y_{D}}{x_{D}+k_{D} y_{D}} .
\end{aligned}
$$

This angle can be obtained by simplification as follows:

$$
\begin{aligned}
\sin \alpha_{D} & =\frac{R_{C}}{R_{D}} \sin \beta_{C D} \\
& =\frac{R_{B} n_{A}}{R_{D} n_{D}} \sin \alpha_{B} .
\end{aligned}
$$

The refraction angle $\beta_{D}$ of point $D$ can be obtained by the refraction law $n_{D} \sin \alpha_{D}=n_{T} \sin \beta_{D}$. Consider

$$
\begin{aligned}
\sin \beta_{D} & =\frac{n_{D}}{n_{T}} \sin \alpha_{D} \\
& =\frac{R_{B} n_{A}}{R_{D} n_{T}} \sin \alpha_{B}
\end{aligned}
$$

or

$$
\tan \beta_{D}=\frac{\sin \alpha_{B}}{\left(R_{D} n_{T} / R_{B} n_{A}\right)^{2}-\sin ^{2} \alpha_{\mathrm{B}}} .
$$


4.7. Total Deflection Angle. As shown in Figure 1, the total deflection angle from incident point $A$ to emergent point $H$ with the residual liquid film is

$$
\eta=\pi-2\left(\beta_{D}-\gamma_{D}-\gamma_{A}\right) .
$$

The following have already been calculated at this point:

$$
\begin{gathered}
\beta_{D}=\arctan \frac{\sin \alpha_{B}}{\left[\left(R_{D} n_{T} / R_{B} n_{A}\right)^{2}-\sin ^{2} \alpha_{B}\right]^{1 / 2},} \\
\gamma_{D}=\arctan \left(\left(\sin \beta_{C D}-\tan \left(\beta_{C D}-\gamma_{C}\right)\right.\right. \\
\left.\cdot\left[\left(\frac{R_{D}}{R_{C}}\right)^{2}-\sin ^{2} \beta_{C D}\right]^{1 / 2}\right) \\
\left.\cdot\left(\tan \left(\beta_{C D}-\gamma_{C}\right) \cdot \sin _{C D} \beta^{2}\right)^{1 / 2}\right), \\
\left.+\left[\left(\frac{R_{D}}{R_{C}}\right)^{2}-\sin ^{2} \beta_{C D}\right]^{-1}\right)
\end{gathered}
$$

$\gamma_{A}$

$$
=\arctan \frac{\sin \alpha_{B} \cdot\left\{\left[\left(R_{A} / R_{B}\right)^{2}-\sin ^{2} \alpha_{B}\right]^{1 / 2}-\cos \alpha_{B}\right\}}{\cos \alpha_{B} \cdot\left[\left(R_{A} / R_{B}\right)^{2}-\sin ^{2} \alpha_{B}\right]^{1 / 2}+\sin ^{2} \alpha_{B}} .
$$

4.8. Constraints of the Model. When a residual liquid film exists on the wall, the constraints must be considered to avoid the total reflection of light as in the simplified model. In this situation, the total reflection of light is likely to occur at point $D$; therefore, the condition of avoiding the total reflection is

$$
\sin \alpha_{D}<\frac{n_{T}}{n_{D}}
$$

or

$$
\sin \alpha_{B}<\frac{R_{D} n_{T}}{R_{B} n_{A}} .
$$

\section{Results and Discussion}

Figure 3 shows the calculated results of total deflection angle $\eta_{D}$ for transformer oil, total deflection angle $\eta_{T}$ for the vacuum without a residual oil film, and total deflection angle $\eta_{i}$ for the vacuum with residual oil films of different thickness $\left(\eta_{1}\right.$ for $\delta=0.05 \mathrm{~mm}, \eta_{2}$ for $\delta=0.1 \mathrm{~mm}$, and $\eta_{3}$ for $\delta=$ $0.15 \mathrm{~mm}$ ). The denser light medium in the tube is a type of industry transformer oil with $n_{D}=1.46$, whereas the thinner light medium in the tube is a vacuum with $n_{T}=1$. The other structure parameters are $R_{A}=12 \mathrm{~mm}, R_{B}=6 \mathrm{~mm}$, and $R_{C}=1.5 \mathrm{~mm}$. The difference between $\eta_{T}$ and $\eta_{i}$ shows the effect of the residual oil film and explains the missing emergent light at point $H_{T}$.

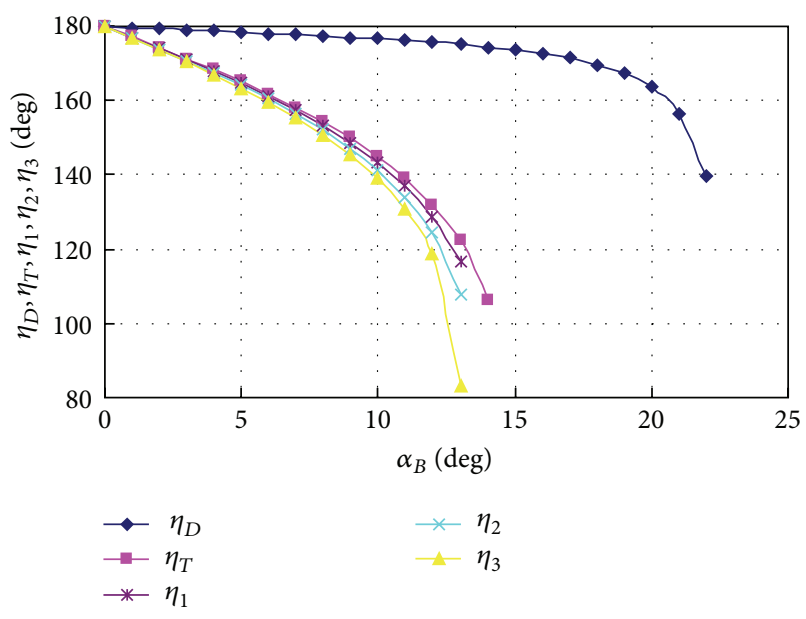

Figure 3: Variation in $\eta_{D}, \eta_{T}$, and $\eta_{i}$ with incident angle $\alpha_{B}$, for example.

5.1. Influence of Liquid Film Thickness. Total deflection angle $\eta$ with the residual liquid film on the wall, which is calculated with (21), is always less than total deflection angle $\eta_{T}$ without the residual liquid film calculated with the equation in [33]. As shown in Figure 3, the thicker the film, the greater the difference between $\eta_{T}$ and $\eta$. Thus, deflection angle difference $\Delta \eta^{\prime}=\eta_{D}-\eta$ between optical thickness and the thinner media is large. When the thickness of the residual liquid film decreases, the light at emergent point $H$ is near point $H_{T}$, and $\eta$ is close to $\eta_{T}$.

5.2. Influence of the Incident Angle. As shown in Figure 3, the larger initial detecting light incident angle $\alpha_{B}$ of detecting light is, the greater the difference between $H$ and $H_{T}$ is under the condition of similar liquid film thickness; this phenomenon is accompanied by the increase in gas-liquid media deflection angle difference $\Delta \eta^{\prime}=\eta_{D}-\eta$. A critical incidence angle $\alpha_{B K}=\arcsin \left(\left(R_{D} n_{T}\right) /\left(R_{B} n_{A}\right)\right)$ is determined with (24) for each specified film thickness. The determined critical incidence angle is always smaller than critical incidence angle $\alpha_{B T \max }=\arcsin \left(\left(R_{C} n_{T}\right) /\left(R_{B} n_{A}\right)\right)$ which does not consider the residual liquid film. When actual incidence angle $\alpha_{B}<\alpha_{B K}$, actual total deflection angle $\eta$ can be calculated with (21). When $\alpha_{B K}<\alpha_{B}<\alpha_{B T \text { max }}$, the total detecting light reflection occurs on the liquid film interface, and the emergent light is not emitted at gas emergent point $H_{T}$ until the liquid film disappears.

5.3. Critical Incidence Angle at Point B. The analysis above provides a very important conclusion: in the presence of residual liquid film on the tube wall, the receipt of the emergent light at emergent point $H_{T}$ is unreliable as mentioned in the simplified model. The best substitution method in this situation is to select $H_{D}$ as the light emergent point, which corresponds to the liquid in the glass tube. Total deflection angle $\eta$ can be calculated with (21). Thus, the effect of the residual liquid film on emergent points $H$ and $H_{T}$ need not be considered even with regard to the possibility of 
the occurrence of total reflection when gas is in the tube. The only requirement is to ensure that the liquid in tube is transparent and that total reflection does not occur when the detecting light passes through the liquid. Therefore, the maximum allowable incident angle (critical incidence angle) at point $B$ is

$$
\alpha_{B D \max }=\arcsin \left(\frac{n_{D} R_{C}}{n_{A} R_{B}}\right) .
$$

\section{Conclusions}

The optical fiber liquid level sensor composed of a glass tube level gauge and a fiber optic sensor can monitor transparent liquid level in a thin glass tube through the method based on the light refraction principle. The acceptance fiber should be set at the emergent point of the optically denser medium in the tube when the influence of the residual liquid film on the tube wall cannot be ignored to receive the emergent light signal. The total reflection of the detecting light in the optically thinner medium can generally be adopted by increasing the incident angle to within the constraint condition of the optically denser medium.

\section{Conflict of Interests}

The authors declare that there is no conflict of interests regarding the publication of this paper.

\section{Acknowledgments}

This research was supported by National Natural Science Foundation of China (Grants nos. 31000665, 51176027, 31371873, and 31300408) and the Fundamental Research Funds for the Central Universities of China (Grant no. N130403001).

\section{References}

[1] T. A. Hughes, Measurement and Control Base, Instrumentation System, 2002.

[2] I. D. Velt and Y. D. Mikhailova, "An electromagnetic method of measurement of the flow rate and level of a liquid in free-flow pipelines," Measurement Techniques, vol. 56, no. 7, pp. 797-804, 2013.

[3] K. Matsumoto, M. Sobue, K. Asamoto, Y. Nishimura, S. Abe, and T. Numazawa, "Capacitive level meter for liquid hydrogen," Cryogenics, vol. 51, no. 2, pp. 114-115, 2011.

[4] J. Liu, X. Z. Chen, and Z. Zhang, "A novel algorithm in the FMCW microwave liquid level measuring system," Measurement Science \& Technology, vol. 17, no. 1, pp. 135-138, 2006.

[5] V. E. Sakharov, S. A. Kuznetsov, B. D. Zaitsev, I. E. Kuznetsova, and S. G. Joshi, "Liquid level sensor using ultrasonic Lamb waves," Ultrasonics, vol. 41, no. 4, pp. 319-322, 2003.

[6] E. Musayev and S. E. Karlik, "A novel liquid level detection method and its implementation," Sensors and Actuators A: Physical, vol. 109, no. 1-2, pp. 21-24, 2003.

[7] S. Khaliq, S. W. James, and R. P. Tatam, "Fiber-optic liquid-level sensor using a long-period grating," Optics Letters, vol. 26, no. 16, pp. 1224-1226, 2001.
[8] S. C. Bera, J. K. Ray, and S. Chattopadhyay, "A low-cost noncontact capacitance-type level transducer for a conducting liquid," IEEE Transactions on Instrumentation and Measurement, vol. 55, no. 3, pp. 778-786, 2006.

[9] N. B. Manik, S. C. Mukherjee, and A. N. Basu, "Studies on the propagation of light from a light-emitting diode through a glass tube and development of an optosensor for the continuous detection of liquid level," Optical Engineering, vol. 40, no. 12, pp. 2830-2836, 2001.

[10] B. Xu, J.-Q. Li, Y. Li, and X.-Y. Dong, "A thin-core fiber modal interferometer for liquid-level sensing," Chinese Physics Letters, vol. 29, no. 10, Article ID 104209, 2012.

[11] C.-L. Lee, Y.-C. Zheng, C.-L. Ma, H.-J. Chang, and C.-F. Lee, "Dynamic micro-air-bubble drifted in a liquid core fiber FabryPérot interferometer for directional fiber-optic level meter," Applied Physics Letters, vol. 102, no. 19, Article ID 193504, 2013.

[12] X. P. Zhang, W. Peng, Z. G. Liu, and Z. F. Gong, "Fiber optic liquid level sensor based on integration of lever principle and optical interferometry," IEEE Photonics Journal, vol. 6, no. 2, 2014.

[13] J. E. Antonio-Lopez, D. A. May-Arrioja, and P. Likamwa, "Fiberoptic liquid level sensor," IEEE Photonics Technology Letters, vol. 23, no. 23, pp. 1826-1828, 2011.

[14] F. Pérez-Ocón, M. Rubiño, J. M. Abril, P. Casanova, and J. A. Martínez, "Fiber-optic liquid-level continuous gauge," Sensors and Actuators, A: Physical, vol. 125, no. 2, pp. 124-132, 2006.

[15] P. Raatikainen, I. V. Kassamakov, R. D. Kakanakov, and M. Luukkala, "Fiber-optic liquid-level sensor," Sensors and Actuators, A: Physical, vol. 58, no. 2, pp. 93-97, 1997.

[16] K.-R. Sohn and J.-H. Shim, "Liquid-level monitoring sensor systems using fiber Bragg grating embedded in cantilever," Sensors and Actuators, A: Physical, vol. 152, no. 2, pp. 248-251, 2009.

[17] J. E. Antonio-Lopez, J. J. Sanchez-Mondragon, P. LiKamWa, and D. A. May-Arrioja, "Fiber-optic sensor for liquid level measurement," Optics Letters, vol. 36, no. 17, pp. 3425-3427, 2011.

[18] S. M. Chandani and N. A. F. Jaeger, "Optical fiber-based liquid level sensor," Optical Engineering, vol. 46, no. 11, Article ID 114401, 2007.

[19] S. Binu, K. Kochunarayanan, V. P. M. Pillai, and N. Chandrasekaran, "PMMA (polymethyl methacrylate) fiber optic probe as a noncontact liquid level sensor," Microwave and Optical Technology Letters, vol. 52, no. 9, pp. 2114-2118, 2010.

[20] P. Nath, P. Datta, and K. C. Sarma, "All fiber-optic sensor for liquid level measurement," Microwave and Optical Technology Letters, vol. 50, no. 7, pp. 1982-1984, 2008.

[21] H. Golnabi, "Design and operation of a fiber optic sensor for liquid level detection," Optics and Lasers in Engineering, vol. 41, no. 5, pp. 801-812, 2004.

[22] V. A. Svirid, V. De Leon, and S. N. Khotiaintsev, "A prototype fiber-optic discrete level-sensor for liquid propane-butane," IEICE Transactions on Electronics, vol. E83-C, no. 3, pp. 303307, 2000.

[23] D. Sengupta, M. Sai Shankar, P. Saidi Reddy, R. L. N. Sai Prasad, K. S. Narayana, and P. Kishore, "Micro-change in liquid level sensing by extrinsic fiber optic sensor," Optoelectronics and Advanced Materials, Rapid Communications, vol. 5, no. 5, pp. 491-494, 2011.

[24] I. K. Ilev and R. W. Waynant, "All-fiber-optic sensor for liquid level measurement," Review of Scientific Instruments, vol. 70, no. 5, pp. 2551-2554, 1999. 
[25] P. Raatikainen, I. V. Kassamakov, R. D. Kakanakov, and M. Luukkala, "Fiber-optic liquid-level sensor," Sensors and Actuators A: Physical, vol. 58, no. 2, pp. 93-97, 1997.

[26] B. Gu, W. Qi, Y. Zhou, Z. Wu, P. P. Shum, and F. Luan, "Reflective liquid level sensor based on modes conversion in thin-core fiber incorporating titled fiber Bragg grating," Optics Express, vol. 22, no. 10, pp. 11834-11839, 2014.

[27] P. Nath, H. K. Singh, D. Tiwari, and T. Basumatry, "Fiberoptic liquid level sensor based on coupling optical path length variation," Review of Scientific Instruments, vol. 83, no. 5, Article ID 055006, 2012.

[28] X. Lin, L. Ren, Y. Xu et al., "Low-cost multipoint liquid-level sensor with plastic optical fiber," IEEE Photonics Technology Letters, vol. 26, no. 16, pp. 1613-1616, 2014.

[29] H. P. Gong, H. F. Song, S. L. Zhang, K. Ni, and X. Y. Dong, "An optical liquid level sensor based on polarization-maintaining fiber modal interferometer," Sensors and Actuators A: Physical, vol. 205, pp. 204-207, 2014.

[30] D. Sengupta and P. Kishore, "Continuous liquid level monitoring sensor system using fiber Bragg grating," Optical Engineering, vol. 53, no. 1, Article ID 017102, 2014.

[31] T. Lu, Z. Li, D. Xia, K. He, and G. Zhang, "Asymmetric FabryPerot fiber-optic pressure sensor for liquid-level measurement," Review of Scientific Instruments, vol. 80, no. 3, Article ID 033104, 2009.

[32] W. H. Wang and F. Li, "Large-range liquid level sensor based on an optical fibre extrinsic Fabry-Perot interferometer," Optics and Lasers in Engineering, vol. 52, no. 1, pp. 201-205, 2014.

[33] W. Liang, S. Zhang, and G. Ji, "A novel fiber-optic liquid level sensor with adjustable light path based on principle of light refraction," Applied Mechanics and Materials, vol. 26-28, pp. 573-578, 2010. 

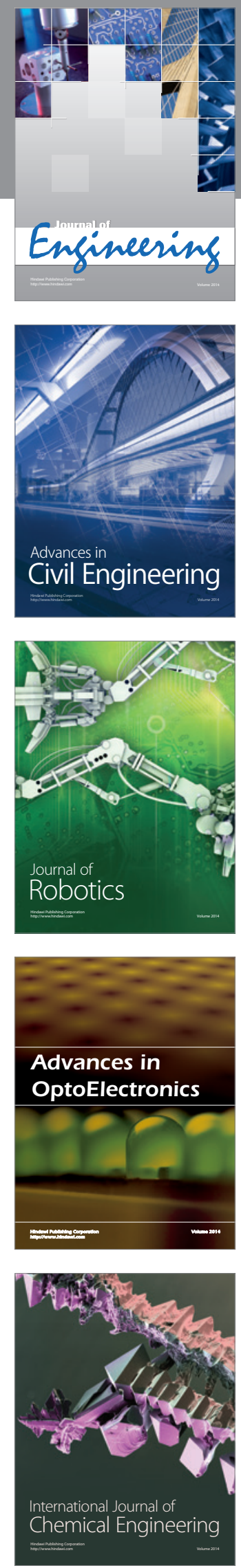

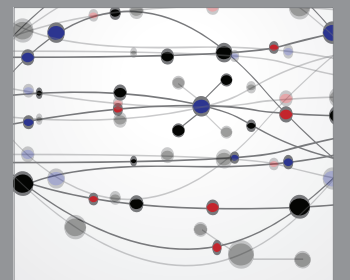

The Scientific World Journal
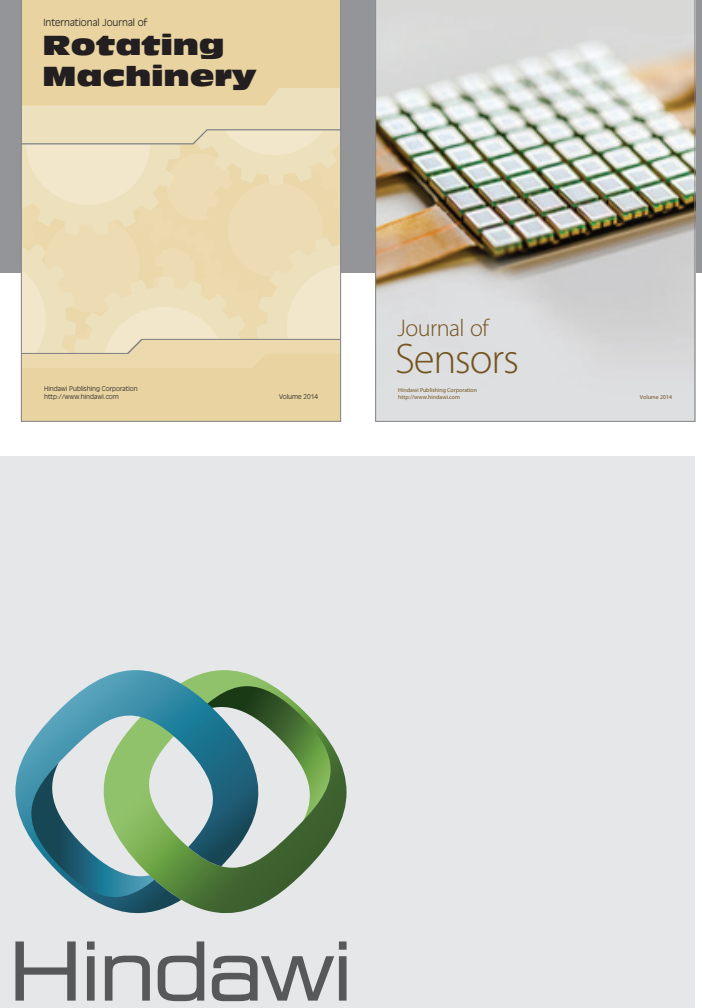

Submit your manuscripts at http://www.hindawi.com
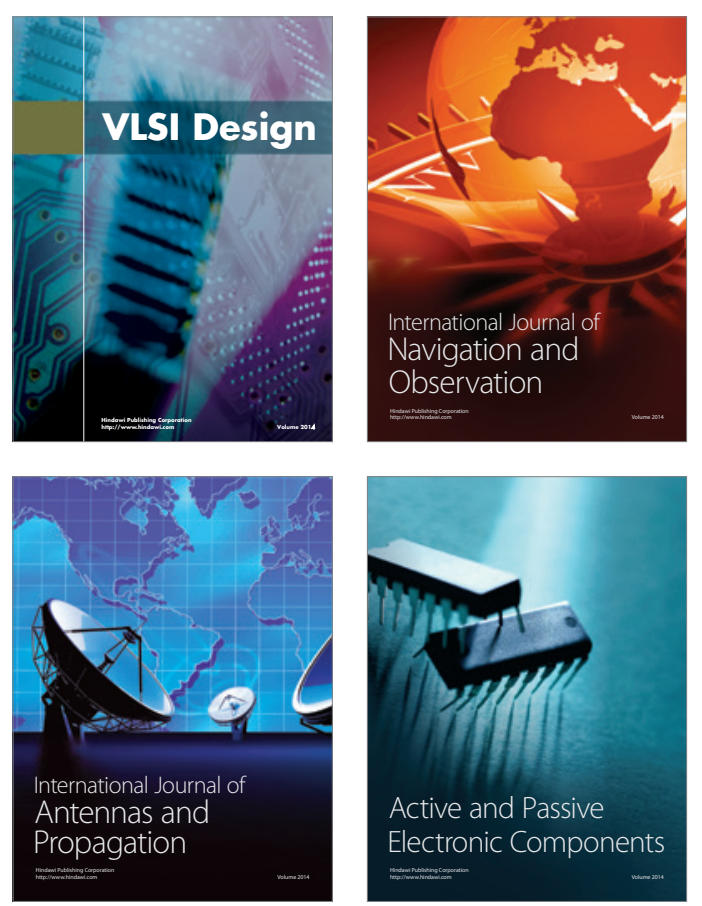
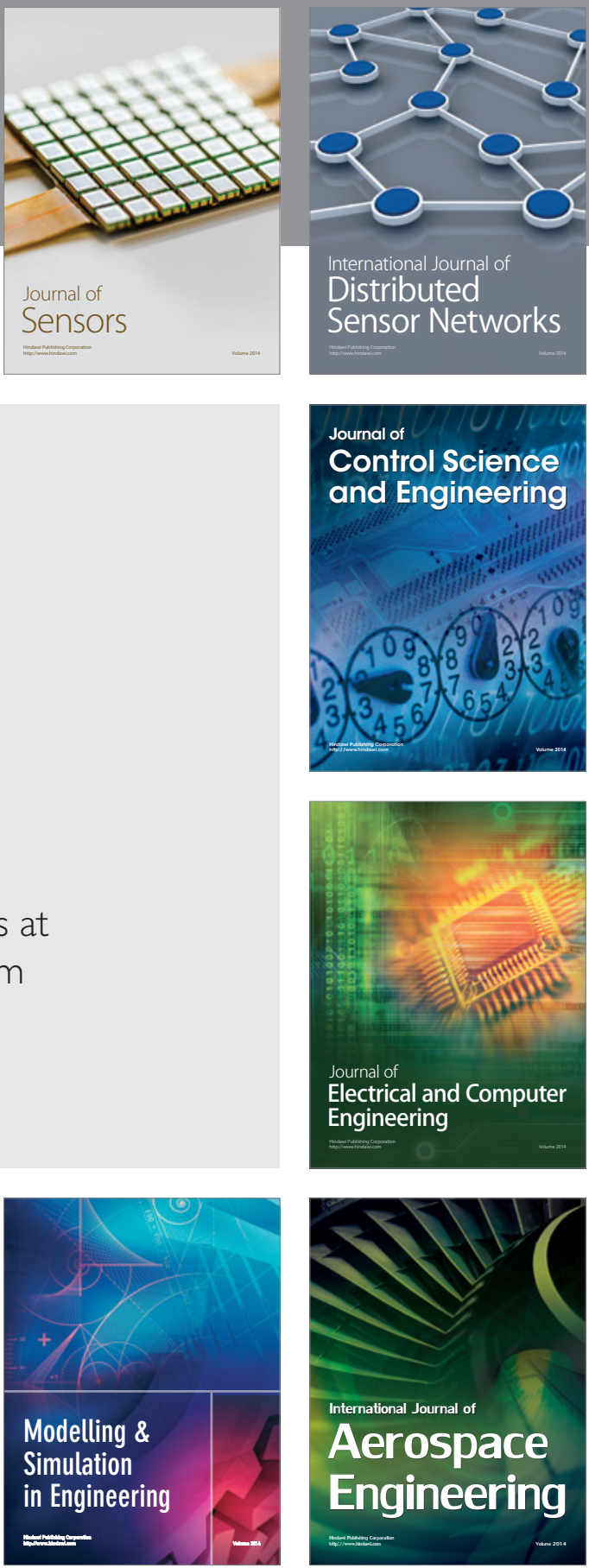

Journal of

Control Science

and Engineering
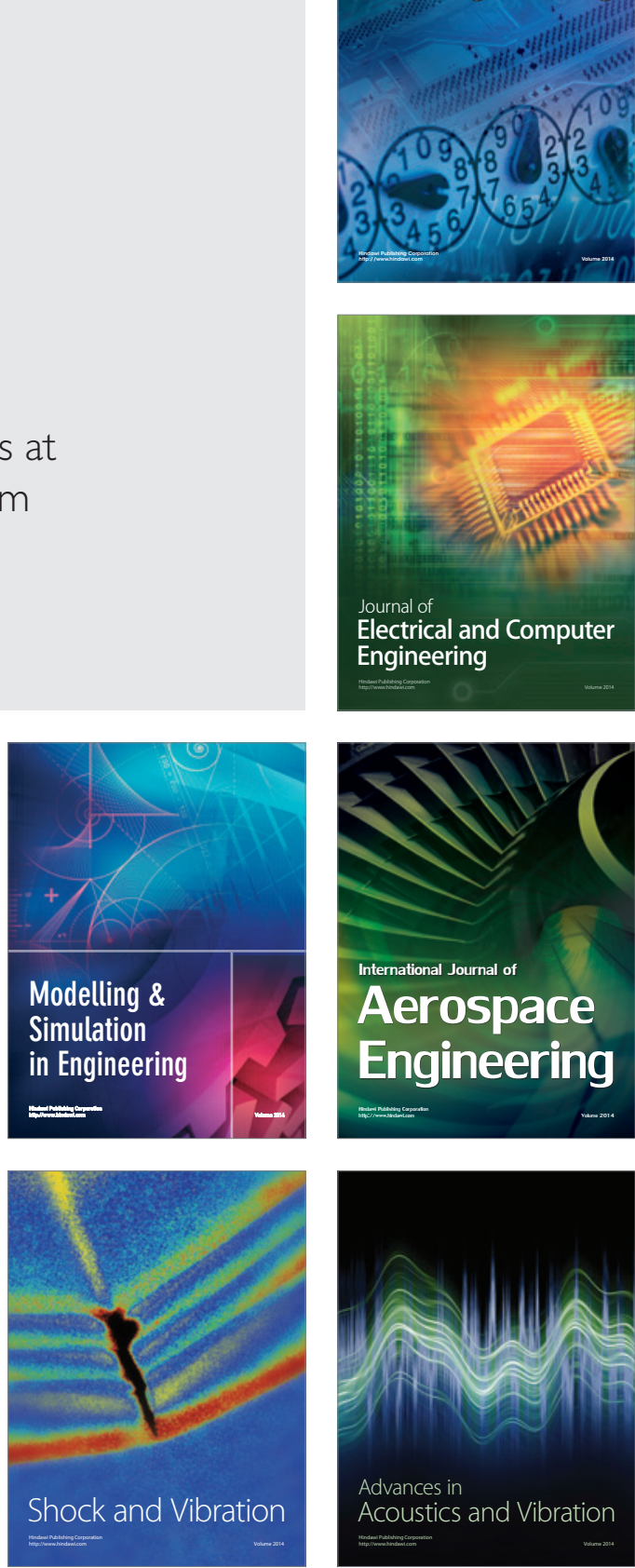